\title{
The Sizable Difficulty in Recognizing Unfamiliar Faces Differing Only Moderately in Orientation in Depth is a Function of Image Dissimilarity
}

\author{
Catrina M. Hacker ${ }^{1}$, Irving Biederman ${ }^{1,2^{*}}$, Tianyi Zhu², Miles Nelken ${ }^{1}$, Emily X. Meschke ${ }^{1}$ \\ ${ }^{1}$ Program in Neuroscience, ${ }^{2}$ Department of Psychology, University of Southern California \\ *Corresponding author: bieder@usc.edu
}

Attempting to match unfamiliar faces at moderate differences in orientation in depth is surprisingly difficult. No general account of these costs has been offered. We assessed the effects of orientation disparity in a match-to-sample paradigm of a triangular display of three faces. Two lower test faces, a matching face and a foil, were always at the same orientation and differed by $0^{\circ}$ to $20^{\circ}$ from the sample on top. The similarity of the images was scaled by a model based on simple cell tuning that correlates almost perfectly with psychophysical similarity. Two measures of face similarity accounted for matching performance: a) the decrease in similarity between the images of the matching and sample faces produced by increases in their orientation disparity, and b) the similarity between the matching face and the selection of a particular foil. The two images of the same face at a $20^{\circ}$ difference in orientation revealed a previously unappreciated marked increase in dissimilarity that was so high that it could be equivalent to the image dissimilarity between two faces at the same orientation, but differing in race, sex, and expression. The $20^{\circ}$ orientation disparity was thus sufficient to yield a sizeable $301 \mathrm{msec}$ increase in reaction time.

Keywords: Face Recognition; Matching Depth-Rotated Faces; Gabor-jet model; Face similarity; Match-to-sample.

\section{INTRODUCTION}

One remarkable feature of face recognition is that familiar faces can be recognized from various viewpoints despite the large distortions of the 2D retinal projections of the face produced by the variations in orientation. Conversely, in the absence of salient distinguishing local features, recognition of unfamiliar faces has consistently been shown to incur large costs when recognition or matching has to be achieved even over moderately different orientations in depth ${ }^{1-9}$. Somewhat surprisingly, there has been little explanation as to why the disparity in the orientation of faces produces such sizeable costs. 
The present study employed a minimal match-to-sample task in which subjects viewed a triangular display of three computer-generated faces (Fig. 1) and attempted to select which one of two lower test faces was identical in identity to the upper face. On some trials, the faces could all be at the same orientation, in which case the image of the matching test face was identical to the sample. On other trials, the test faces differed in orientation in depth from the sample which meant that the images of the sample and the correct test face differed, although the identity was the same. The test face differing in identity from the sample served as the foil and could vary in similarity to the matching face but was always at the identical orientation to the matching face.

Some studies of the costs of orientation disparity on face recognition have used multiple foils ${ }^{1,2}$ typically held in memory, rendering it difficult, if not impossible to isolate an effect of distractor similarity. By employing only a single distractor which was in view during the matching, the present paradigm allowed a quantitative assessment of distractor similarity on the matching of face percepts rather than the memory of those percepts. The matching of faces at different disparities in depth could be separated into two quantitative measures of similarity between pairs of faces: a) The dissimilarity between the sample and matching face produced by orientation disparity, and b) the similarity of the foil to the matching test face, dependent on the particular face selected as a foil on that particular trial, which determined the discrimination challenge. The two test faces-matching and foil-were always at the same orientation in depth which might or might not match the orientation of the sample. The greater the dissimilarity of the sample to the matching face and the smaller the dissimilarity between matching face and foil, the greater the expected difficulty in selecting the correct test face. In the absence of a principled measure of the similarity of faces, these two quantities had never, heretofore, been evaluated, either individually or in concert.

The Gabor-jet model ${ }^{10,11}$ provides a means for scaling the similarity of images of faces based on a model of V1 simple cell filtering. The speed and accuracy of matching faces that are all at the same orientation is almost perfectly predicted by the similarity values of the model, with correlations with error rates in the mid $.90 \mathrm{~s}$, even without a correction for the unreliability of the behavioral data. ${ }^{12}$ Justification for the Gabor-jet scaling of the similarity of faces, beyond 
its excellent predictability of psychophysical face similarity, derives from experiments ${ }^{13}$ showing that the representation of faces in FFA, a cortical area critical for individuating faces $^{14,15}$ is highly sensitive to the specific spatial (Fourier) kernels specifying the orientation, scale, and position of contrast that distinguish one image of a face from another. It is the image similarity rather than the extraction of the underlying 3D representation of a face that is relevant to psychophysical matching. Thus, the advantage of matching bilaterally symmetrical faces is reduced when the faces are illuminated by asymmetrical lighting. ${ }^{15}$

The design of the present study employed a scaling of the dissimilarity between the matching test face and the sample as well as the matching test face and the foil (which were always at the same orientation). This allowed a test of whether the model's similarity values would also be highly predictive of performance when the faces were at different orientations in depth. In the absence of a principled measure of face similarity, past attempts at explanations of face rotation costs typically interpreted the costs in terms of viewing angle per se. ${ }^{4}$ This implicitly assumes a "protractor-in-the-head" representation in which matching is achieved through mental rotation or an alignment of a subset of features of one image to a subset of features of another stimulus. However, with a quantitative measure of face similarity one can a) assess the extent to which matching speed and accuracy is a positive function of the overall similarity of the matching test face to the sample and b) a negative function of the similarity of the foil to the matching stimulus, without a commitment to a particular angular transformation (which is difficult to implement in the absence of distinguishing local features to serve as landmarks). The investigation assessed the extent to which these two measures of image dissimilarity could account for human performance in matching faces differing in orientation.

One advantage of the match-to-sample paradigm over the oft used same-different judgment task is that subjects do not have to adopt an arbitrary criterion as to whether two highly similar faces are identical or not. Rather, a relative criterion-Which face more closely resembles the sample?-suffices. This criterion can be adopted because, unlike memory tasks with more than one possible face, the foil is well defined and in view so the similarity of the foil to the matching face can be calculated and its effects on performance evaluated. By using computer generated faces, the presence of local, distinguishing features that are abundant in 
photographs, such as a beauty mark, blemish, or the configuration of eyebrow hairs, could be excluded. The absence of such features meant that face matching in the present investigation required perception and discrimination of subtle metrically-varying configurations of attributes, such as the height of the cheekbones and the precise positions and shapes of the eyes, nose, and mouth.

\section{METHODS}

Stimuli. The faces were created using FaceGen Modeller (Singular Inversions, Toronto, Canada), a 3D face modeling program. The core image was that of a bald, 20-year-old, Caucasian, gender neutral individual on a black background (Figure 1). Twenty different identities were generated by varying the distances between eyes, nose, and mouth; height/prominence of cheek bones; jaw width; and very slightly varying the length and width of face parts such as the eyes, nose, and mouth (Figure 2). The faces could be rotated $0^{\circ}, 13^{\circ}$, or $20^{\circ}$ in depth. The variations were metric, such as the degree of curvature of the eyebrows, rather than qualitative (or nonaccidental), such as whether eyebrows were curved or straight. These subtle variations of the default face were made to render the differences between the faces largely ineffable as occurs with naturally similar faces. ${ }^{3}$ The computer generation of the faces excluded the presence of local distinguishing features, such as beauty marks or moles, which would have allowed the subjects to zoom in and employ such features for distinguishing the faces rather than processing the whole face. The generation of the faces also avoided noticeable differences in standard population-defined categories such as sex, race, age, expression or attractiveness which could have been employed to select a response. All the stimuli were in grey scale and were $256 \times 256$ pixels in extent.

Of a possible 190 combinations of matching-foil pairs of faces (disregarding status as matching or foil), only the 180 combinations with a normalized dissimilarity value of 1.50 or greater were used. The Gabor values are normalized by dividing by the number of jets, 100, that the scaling employed in this investigation. Each of these pairs of faces appeared twice throughout the experiment: once with the first face as the sample and a second time with the second face as the sample, for a total of 360 trials. The dissimilarity values between the 
matching and distractor faces were divided into four bins with an equal number of trials in each bin.
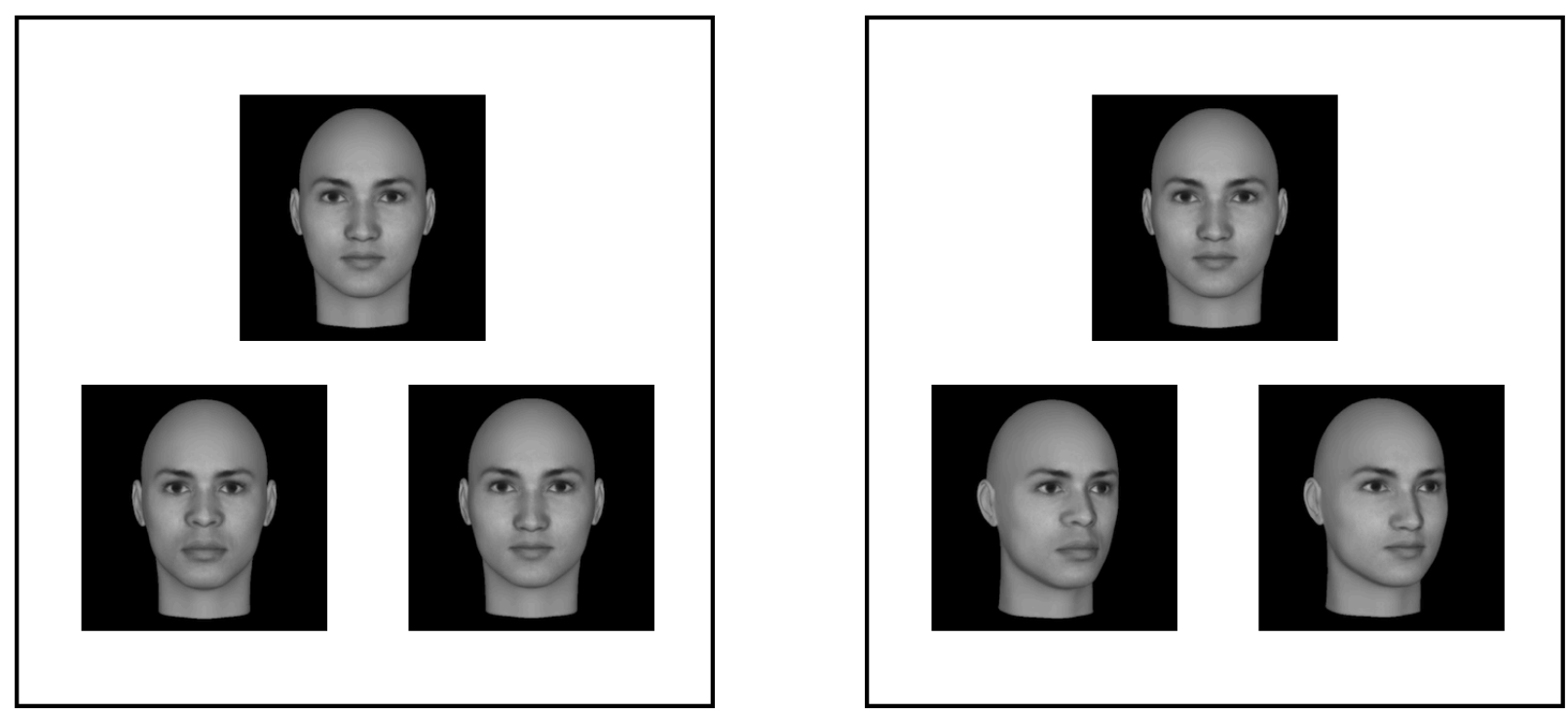

Figure 1. Sample displays from two different trials with identical sample and test faces. Left panel: An example of the match-to-sample task with the sample (top) and the two test faces (bottom) presented at the same frontal $\left(0^{\circ}\right)$ orientation. Right panel: An example of a $20^{\circ}$ trial in which the same test faces from the left panel differ from the same $\left(0^{\circ}\right)$ sample face (top) by $20^{\circ}$. The reader may sense the increased difficulty in matching when the sample and test faces are at disparate orientations in depth as the identities are the same in both panels. Both $0^{\circ}$ and rotated trials (either $13^{\circ}$ or $20^{\circ}$ ) could have the sample at any of the three orientations $\left(0^{\circ}, 13^{\circ}\right.$, or $20^{\circ}$ ). The left-right designation of the stimuli refers to the viewer's (rather than the head's) left-right orientation. The normalized Gabor dissimilarity between the matching and foil faces was 2.74 for the left panel and 2.93 for the right panel. The Gabor dissimilarity between the matching test face and the sample is 0 in the left panel and 5.57 in the right panel. In both cases the correct match to the sample is on the right and the foil is on the left.

Design and Procedure. Subjects performed the task which consisted of 360 2AFC match-tosample trials on testable.org. On each trial, subjects viewed a triangular arrangement of three faces with one face (the sample) centered above two lower faces (the test stimuli), one of which matched the identity of the sample (Figure 1). The orientation in depth of the test faces could differ from the sample face by $0^{\circ}, 13^{\circ}$, or $20^{\circ}$. The two test faces were always at the same orientation in depth. Because the orientation in depth of the test stimuli could differ from the sample, the image of the matching face could differ from the sample, but the identity was always an exact match. For trials where the sample and test faces differed in orientation, the 
departure from the $0^{\circ}$ orientation could be implemented in the sample or the test faces, e.g., the sample could be at $0^{\circ}$ and the two test faces at $13^{\circ}$, or the sample could be presented at $13^{\circ}$ and the two faces could be at $0^{\circ}$. In either case, the test faces would be rotated $13^{\circ}$ from the sample (an orientation disparity of $13^{\circ}$ ) and were so classified. Faces were always rotated to their left (subject's right) as in the right panel of Fig. 1. Subjects indicated which of the two test faces matched the identity of the sample by pressing the left or right arrow key as quickly and as accurately as possible. The orientations disparities were approximately balanced over the various levels of matching and foil similarity values.

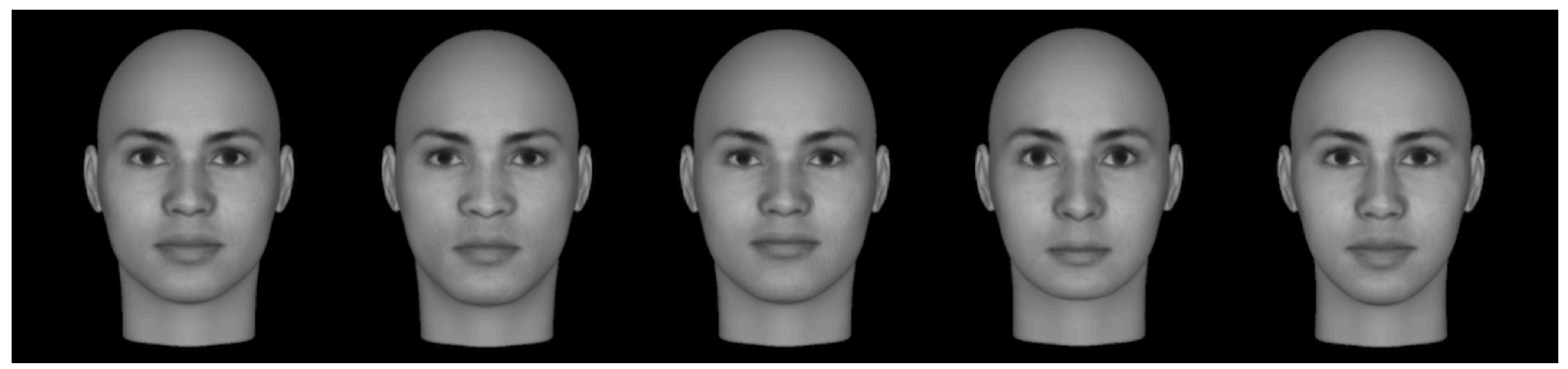

Figure 2. Five examples of the 20 experimental faces. Subtle variation in the features created slightly different appearing individuals. The normalized Gabor dissimilarity ${ }^{9}$ between faces 1 and 2 is 1.49 and between 1 and 5 is 3.63 .

The stimuli were displayed for 5 seconds, although responses were recorded after the cessation of the display on the rare occasion that response time exceeded 5 seconds and the next trial had not yet started. Reaction times that were shorter than $500 \mathrm{msec}$ or longer than $10.0 \mathrm{~s}$ were not included in the data analysis. Within and across each block, the stimuli were balanced by face identity (one of the 20 faces that differed in underlying shape), Gabor dissimilarity between 180 combinations of matching and distractor faces, and orientation condition (e.g., $0^{\circ}-0^{\circ}, 0^{\circ}-13^{\circ}, 0^{\circ}-20^{\circ}, 13^{\circ}-13^{\circ}$, etc.), for which there were 60 trials per condition (the $20^{\circ}-20^{\circ}$ condition was not used). All subjects viewed the same stimuli presented in different random orders. Subjects could pause at their leisure between any of the five blocks, although not between individual trials within a block. The total time for testing was approximately 25 minutes, which included 5 minutes for instructions.

Displays. To ensure that all images were displayed within the boundaries of the screen independent of the particular computer used by a subject, a calibration procedure was run at the start of the experiment. Each subject used the left and right arrow keys to adjust the length 
of a line on the screen to match the horizontal extent of a standard credit card. Subjects were instructed to sit at a viewing distance of approximately an arm's length from the computer screen. At this distance from a 15" laptop screen, each face was bounded by a square that subtended a visual angle of approximately $5.0^{\circ}$ on each side with a horizontal separation of $0.7^{\circ}$ between the lower two test headshots and a vertical separation of $0.7^{\circ}$ between the test and sample headshots (Figure 1). An important design feature of the display was the diagonal arrangement of the faces which defeats local pixel- or feature-based comparison processes which could be more readily engaged if the faces were aligned vertically or horizontally. Stimulus similarity scaling. The Gabor Jet scaling ${ }^{9}$ of the physical similarity of pairs of faces was computed from a $10 \times 10$ grid centered on each face. (The procedure is illustrated in Margalit et al. ${ }^{11}$ which presents an app for computing the Gabor similarity of faces.) Each node of the grid corresponds to the center of the receptive fields of the kernels of one jet (modeling the orientation and scale tuning of a single, simplified V1 hypercolumn) composed of 80 Gabor filters at 8 equally spaced orientations $\left(22.5^{\circ}\right.$ differences in angle), 5 scales, and 2 phases (sine and cosine). The coefficients of the kernels (with the magnitude representing the activation value of a single simple cell) within each jet were then concatenated to an 8000 -element vector representing each image (100 jets $x 80$ kernels). Image similarity was computed as the Euclidean distance between two 8000-value vectors. When images of two faces are identical, the dissimilarity is zero.

As noted previously, the similarity values calculated in this manner predict human psychophysical similarity in a match-to-sample task for faces at the same orientation in depth almost perfectly. ${ }^{12}$ The Gabor-jet dissimilarity values in the present experiment between matching and foil faces on individual trials at $0^{\circ}$ orientation disparity were selected to fall within a range of 1.50 to 4.12 which, for most subjects, placed them in an intermediate range of difficulty that allowed performance to reflect the experimental variations. The dissimilarity values reflect only the physical differences between a pair of faces as registered by the Gabor kernels and are not sensitive, by themselves, to population-defined categories (PDCs) such as sex, age, expression, race, or attractiveness which can be learned from exposures to samples of faces. Using reverse correlation, a classifier for each of these population-defined categories can 
be calculated from the pattern of activation of the various Gabor-like kernels for these categories. ${ }^{17}$ We note that prosopagnosics can typically identify the PDCs of a face, e.g., that it is of a 20ish year-old Caucasian female, but they fail at individuating a face within its PDC. ${ }^{17}$ Indeed, an acquired prosopagnosic with severe bilateral lesions to cortical face areas OFA and FFA is unable to recognize his wife, siblings, or his own face in a mirror but is normal in distinguishing and describing population-defined attributes of faces. ${ }^{17,18}$ This result suggests that the recognition of population-defined attributes engage different networks than those involved in individuating a face and it is face individuation that is the subject of the present investigation.

The dissimilarities of two kinds of relations between the faces on each trial are of particular relevance: a) the dissimilarity between the matching (correct) test face and the sample, reflecting the difference in orientation, and b) the dissimilarity between the matching and foil test faces. The average dissimilarities over orientation angle are shown in Fig. 3 . The dissimilarity between the matching and sample faces increased markedly with increasing orientation disparities, with most of the increase occurring between $0^{\circ}$ and $13^{\circ}$ with a somewhat smaller increase in dissimilarity between $13^{\circ}$ and $20^{\circ}$. We also considered an alternative scaling method, the Fiducial Point Model (FPM) $)^{19,20}$, in which the individual jets are not centered in a regular grid, with a somewhat arbitrary positioning of jets with respect to face features, but are positioned over specific facial landmarks (termed fiducial points), such as the pupil of the left eye or the tip of the nose. The FPM yielded a similar pattern of dissimilarities as those shown in Fig. 3 using the grid model with some puzzling exceptions in that for some faces there was not a monotonic increase in matching-sample dissimilarity with increasing orientation disparities. In general, the FPM did not provide as good a qualitative fit to the data as the grid model in that it failed to show greater costs with increasing orientation disparities and it failed to reflect the slightly greater similarity of the matching (vs. the foil) face to the sample under rotation. Consequently, all analyses reported here employed the grid model. Later we consider why the explicit locations of facial landmarks encoded in the FPM did not provide as good a match to the data as the grid model. 
A straightforward expectation would be that the difficulty in matching faces (i.e., longer RTs and higher error rates) would increase with increasing dissimilarity between the matching face and the sample. We would also expect that an increase in similarity between matching and foil stimuli would render matching more difficult, resulting in longer RTs and higher error rates. A particularly striking effect apparent in Fig. 3 is that the sizable difference in dissimilarity of about 3.00 Gabor units between matching to sample and foil to sample dissimilarities at $0^{\circ}$ disparity (reflecting that the matching face is identical to the sample whereas the selection of the face that serves as a foil differs by an average of 3.00 Gabor units from the sample), virtually disappears at $13^{\circ}$ and $20^{\circ}$. If the matching of the correct test face to the sample is based on Gabor similarity, the loss of the characteristics that distinguished the matching from the foil faces at the modest orientation disparities suggests that there should be a marked increase in difficulty in judging which of the two test faces matches the sample.

As shown in Fig. 3, unlike the dissimilarity of matching to sample faces, the average dissimilarity between the 20 matching and foil test faces remained relatively constant over rotation angle between sample and test faces as the matching and foil faces were always presented at the same orientation. Nonetheless, within a given orientation there was considerable variation in dissimilarity values among the 180 combinations of different foil and matching faces as reflected in the error bars. This variation allowed a straightforward test of whether the effect of the dissimilarities of the foil to matching faces would be independent of the disparity in orientation between matching and sample faces. 


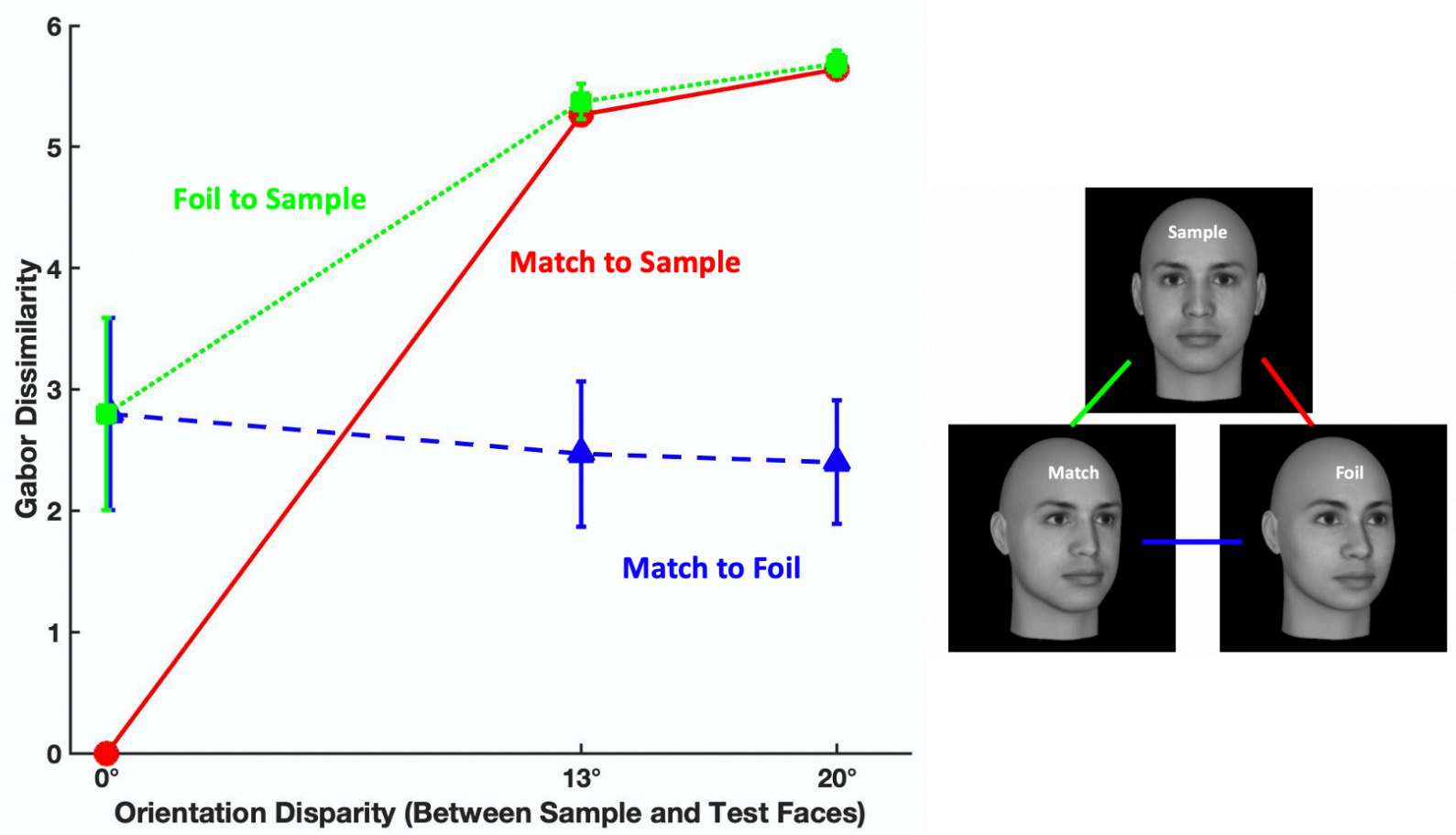

Figure 3. Mean normalized Gabor dissimilarity values for the three stimulus relations on each trial (Match to Sample, Foil to Sample, and Match to Foil) as a function of the orientation difference between the sample and test faces. The matching and foil test faces were always presented at the same orientation. The error bars are the standard deviations of the mean of the Gabor dissimilarity values at each orientation difference between the various instances of the 20 sample and test faces.

Participants. Sixty-five participants (mean age 20.55 yrs., range 18-47 years, 16 males) performed the web-based USC Rotated Face Perception Test, (USC rFPT), for course credit in the Department of Psychology subject pool. Six of these subjects were excluded from further analysis: five had more than 20 correct trials with a reaction time below $750 \mathrm{~ms}$, and one had more than 5 trials with a reaction time greater than 7.5 seconds. Subjects excluded on the basis of fast reaction times all had extremely high error rates, indicative of a lack of engagement in the discriminative challenge. All subjects reported normal or corrected-to-normal vision and no neurological or visual disorders. The work was carried out in accordance with the Code of Ethics of the World Medical Association (Declaration of Helsinki). All subjects gave informed consent in accordance with the procedures approved by University of Southern California's University Park Institutional Review Board. 


\section{RESULTS}

Effect of Orientation Disparity. Figure 4 shows the mean correct RTs as a function of the Match-to-Foil Dissimilarity values separately for the three angular disparities $\left(0^{\circ}, 13^{\circ}\right.$, or $\left.20^{\circ}\right)$ between sample and matching stimuli. For a given orientation disparity, the data are collapsed over the particular orientation disparities between the sample and the matching test faces. Thus, the data for when the sample face was at $0^{\circ}$ and the matching face at $13^{\circ}$ are combined with the data for when the sample face was at $13^{\circ}$ and the matching face at $0^{\circ}$ as there were only small and inconsistent differences in performance when the sample or test faces were rotated. When matching faces at different orientations, subjects appeared more willing to tolerate longer RTs than a higher error rate. An orientation disparity of $20^{\circ}$ produced an increase in error rates of only $3.8 \%$ above that at an orientation disparity of $0^{\circ}$ but a sizeable $301 \mathrm{msec}$ increase in RTs. The greater the orientation difference between matching and sample faces, the greater the difficulty in matching as reflected in longer RTs and higher error rates. For orientation disparities between matching and sample faces of $0^{\circ}, 13^{\circ}$, and $20^{\circ}$, mean RTs (and percent errors) were $2080 \mathrm{msec}$ (14.5\%), $2318 \mathrm{msec}(17.2 \%)$, and $2381 \mathrm{msec}$ (18.3\%), for reaction times $F(2,116)=50.06, p<.001, \eta_{p}{ }^{2}=0.463$, for error rates, $F(2,62)=3.56, p<.05, \eta_{p}^{2}$ $=0.236$. $\left(\eta_{p}{ }^{2}=\right.$ partial eta squared, a measure of the proportion of the total variance associated with an independent variable with the effects of other independent variables and interactions partialed out. ${ }^{20}$ )

Match-to-Foil Dissimilarity Effects. The mean dissimilarity between matching and foil faces remained largely constant as orientation disparity increased, as shown in Fig. 3, so the cost of the disparities in orientation between sample and matching faces was wholly a function of the increased Gabor dissimilarity of the matching test face to the sample as orientation disparity increased. There was, nonetheless, considerable trial-to-trial variation in the match-to-foil similarities among the various pairs of the 20 matching and foil test faces within each orientation disparity. Data were grouped into four bins based on Gabor dissimilarity between matching and foil faces and mean correct RTs were computed for each bin at each orientation disparity as shown in Fig. 4. (These results were highly similar to those obtained with the raw data.) With the exception of the single aberrant point on the $13^{\circ}$ orientation disparity function, 
the Match-to-Foil dissimilarity values were linearly related to RTs with greater dissimilarities (i.e. more easily distinguished faces) yielding shorter $\mathrm{RTs}, F(3,174)=51.90, p<.001, \eta_{p}{ }^{2}=$ 0.472. The point is termed "aberrant" as it had a mean RT that exceeded that of the two matchto-sample points on that same $13^{\circ}$ function that had lower match-to-foil dissimilarity values and even exceeded the corresponding point on the $20^{\circ}$ function which accounted for the shallow slope of the $13^{\circ}$ function and the low correlation with match-to-foil dissimilarity relative to the $0^{\circ}$ and $20^{\circ}$ functions.

The Pearson correlations between the Match-to-Foil (bin) dissimilarity values and RTs were -0.92 for $0^{\circ}$ disparity, -0.61 for $13^{\circ}$ disparity and -0.98 for $20^{\circ}$. Correlations for unbinned data (not shown) showed similar trends to the binned data: $r=-0.44$ for $0^{\circ}$ disparity, $p<0.0001,95 \% \mathrm{Cl}=[-0.573,-0.284], r=-0.15$ for $13^{\circ}$ disparity, $p=0.10,95 \% \mathrm{Cl}=[-0.32,0.03]$, and $r=-0.31$ for $20^{\circ}$ disparity, $p<0.001,95 \% \mathrm{Cl}=[-0.46,-0.14]$. The slope of the correlation between Gabor dissimilarity and RTs (in units of msec/unit of Gabor dissimilarity) was - 0.160 for $0^{\circ}$ disparity, -0.073 for $13^{\circ}$ disparity and -0.158 for $20^{\circ}$ disparity, showing the same trends as the binned data in Figure 4.

The main effect of Orientation Disparity was highly significant, $F(2,116)=154.23, p<$ $.001, \eta_{p}{ }^{2}=0.727$, with the greater the dissimilarity between matching and sample faces (associated with greater angular disparity), the longer the RTs. Given the power in the analysis, the Orientation X Bin interaction was also significant, $F(6,348)=6.05, p<.001$ although the $\eta_{p}{ }^{2}$ value was only 0.094 , classified as a weak effect, attributable to the single point on the $13^{\circ}$ orientation disparity function. The relative magnitude of the effect of the interaction can be appreciated in comparison to the $\eta_{\mathrm{p}}{ }^{2}$ values for the main effects of Orientation and Match-toFoil (bin) dissimilarity which were 7.7 and 5.0 times, respectively, the magnitude of their interaction. 


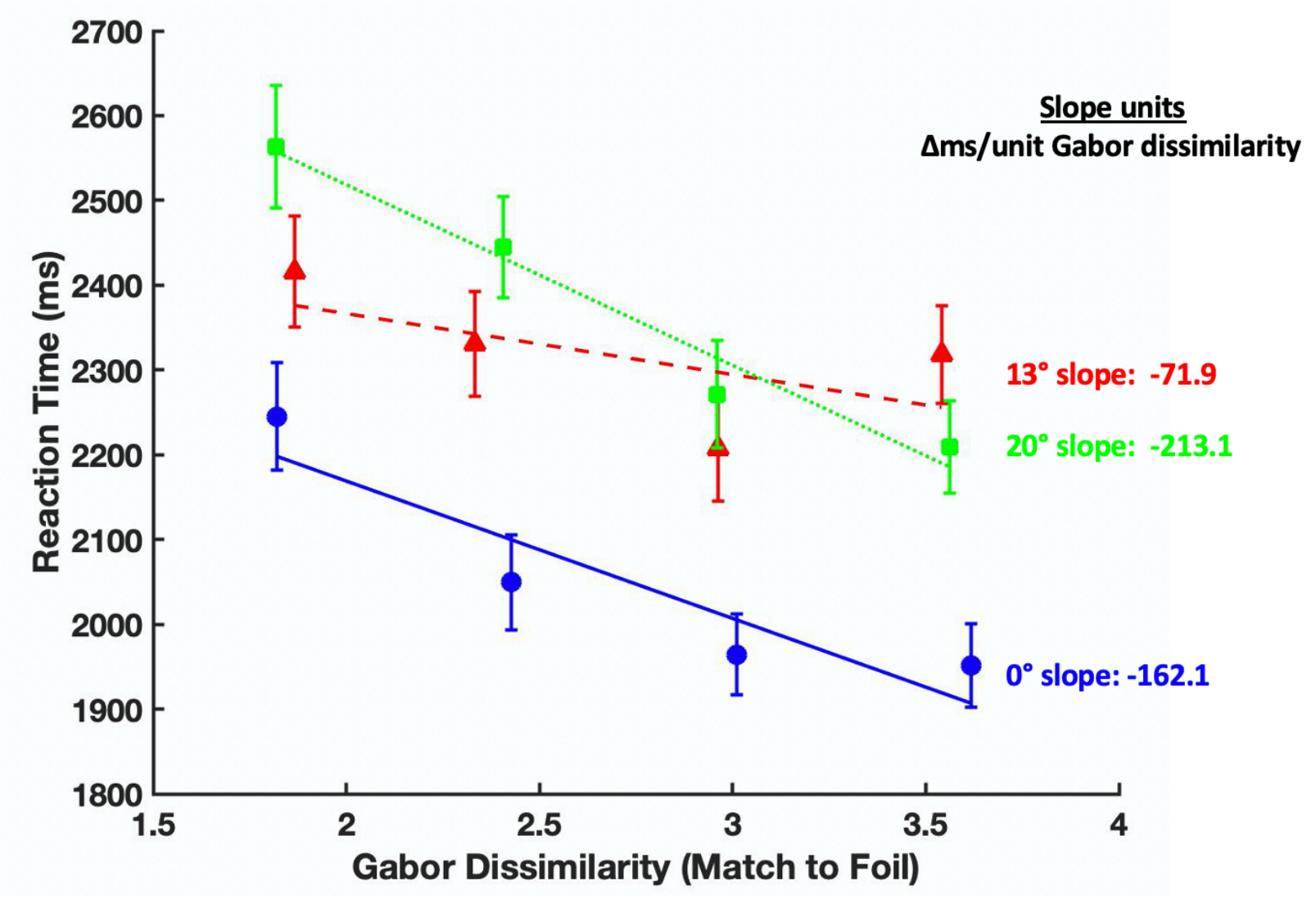

Figure 4. Mean correct reaction time ( $\mathrm{msec}$ ) as a function of the normalized binned Gabor dissimilarity values between the matching and the foil faces over the three levels of orientation disparity. The slopes are in units of msec per unit of normalized Gabor dissimilarity.

The most striking aspects of these data are that the reduced cost (decrease in RTs) is linear with an increase in Gabor dissimilarity between matching and foil faces. This effect is roughly independent of orientation disparity with the shallower slope of the $13^{\circ}$ function attributable to the previously noted single aberrant point at the highest match-to-foil dissimilarity value being a departure from what otherwise would be additivity in the RTs for orientation disparity and Match-to-Foil dissimilarity. To assess the possible role of errors in this pattern of data, the dependent variable in Fig. 4 was plotted as inverse efficiencies ${ }^{22}$ in which each RT was divided by the accuracy (percent correct) at that point (not shown). The picture that emerged was virtually identical to the data in Fig. 4 so differences in error rates are unlikely to be the cause of the shallower slope at $13^{\circ}$. This point is discrepant with the other data in the experiment and the results of prior studies consistently showing that the higher the match-to-foil Gabor dissimilarity, the shorter the RTs. ${ }^{12}$ 
Relative magnitude of the effects of variations in the match-to-sample and foil-tosample similarities. As noted above, performance (RTs) in this task was largely a function of two parameters: a) the similarity of the matching face to the sample, which would decrease with an increase in orientation disparity, and b) the similarity of the foil to the matching stimulus which would be unaffected by orientation disparity but would be a function of the particular faces selected for a given trial.

Compared to the $0^{\circ}$ orientation difference between the matching stimulus and the sample, the rotation of $20^{\circ}$ decreased the similarity between the matching stimulus and the sample by an average of 5.67 Gabor units producing a $301 \mathrm{msec}$ increase in RTs or an increase of 53 msec per unit of reduced Gabor similarity. As shown in Fig. 4, the mean slope of the three functions is $-148 \mathrm{msec} /$ unit of Gabor dissimilarity suggesting that the effect of similarity between the foil and matching stimulus (i.e., the discrimination challenge) is approximately 2.8 times the effect of dissimilarity between the matching test stimulus and the sample. Put another way, for each unit of increased Gabor similarity between matching and foil stimuli, the increase in RTs is 2.8 times greater than the increase in RTs produced by each unit of decreased Gabor similarity produced by the orientation disparity between sample and matching stimulus.

Fiducial point scaling. As noted earlier, an alternative scheme for the similarity scaling of faces is to use a Fiducial Point Model (FPM) in which each jet is centered on a particular face landmark, such as the pupil of the left eye or the tip of the nose. Although the FPM captures the observer's face knowledge as to corresponding face features in two faces at different orientations (e.g., placement of pupils of the eyes, tip of the nose), somewhat surprisingly, the FPM yielded larger Gabor dissimilarities compared to the grid model, without any noticeable gain in predictability. This is likely a consequence of the positioning of the jets in the fiducial model FPM at points of higher contrast variation than the jet locations in the grid model. The latter model has some jets centered on the black background or middle of the cheeks where the change in dissimilarity as the face was rotated in depth, for kernels with smaller receptive fields, would be very low. Of greater concern as to the adequacy of the FPM, for eight of the 20 faces, the FPM yielded smaller Gabor dissimilarities between $0^{\circ}$ and $20^{\circ}$ orientations of those faces compared to the dissimilarities between the $0^{\circ}$ and $13^{\circ}$. In the grid model, all 20 faces 
were more dissimilar to the $0^{\circ}$ face at $20^{\circ}$ compared to $13^{\circ}$, in line with the data showing increased difficulty in matching over a $20^{\circ}$ disparity than a $13^{\circ}$ disparity.

Still another discrepancy of the FPM with the behavioral data was that at an orientation disparity of $13^{\circ}$ it failed to reflect the (slightly) greater similarity of the matching face to the sample compared to the foil with the sample so it could not account for the increase in RTs.

It might seem surprising that the FPM in its explicit coding of aspects of face knowledge does not achieve any gain in predictability compared to the grid model. We propose that what the FPM renders explicit about the face, such as the locations of the pupils of the eyes or the corners of the mouth, are not what limits performance on the present task. We presume that all individuals with normal vision would be able to locate the various face landmarks. The challenge would be in recognizing the extent of the metric deformations in the images of faces when matching had to be executed at different disparities in orientations. The FPM's specification of the locations of facial landmarks would seem to have its greatest value in finding a face in an uncertain location rather than in determining its identification.

\section{DISCUSSION and CONCLUSION}

Although sizable costs in recognizing or matching unfamiliar faces differing modestly in orientation have been reported in the literature, there had been no general explanation of these costs. We document that two stimulus similarity parameters may be sufficient to provide an account of these costs: 1) The dissimilarity of the matching face to the sample produced by the orientation disparity between the two, and 2) the similarity of the foil to the matching face which defines the discrimination challenge. High similarity of the foil and matching stimulus increases the uncertainty as to which test face is to be matched to the sample. As scaled by the Gabor-jet model, increases in the values of each of these parameters produced increases in RTs. Moreover, the effects of the two were approximately additive on RTs (save for the single point on the $13^{\circ}$ function). Insofar as the similarity of the foil to the matching face can be regarded as the perceptual challenge in this task, the approximate additivity with the effects of orientation disparity suggests, by additive factors logic, ${ }^{23,24}$ that the two factors affect different processing stages. 
The relation between the recognition of depth-rotated faces and the recognition of depth-rotated objects. The most significant determinant of the costs in matching or recognizing similar depth-rotated visual entities-faces or objects - is whether the stimuli to be distinguished differ in nonaccidental properties. ${ }^{25,26,27}$ Nonaccidental properties (NAPs) are characteristics or features of a visual entity that are invariant with the orientation of the object in depth. Such properties can be an aspect of shape, such as whether a particular contour, say an eyebrow, is straight or curved, or they can be a characteristic of a surface feature of the object, such as whether there is or is not a dark spot, such as a beauty mark, in a given region of a face. If the stimuli can be distinguished by a NAP (or NAPs) and the object or face is at an orientation where the NAP is in view, then a rapid, correct response can be produced without even processing the face itself. ${ }^{26}$ NAPs are distinguished from metric properties (MPs) such as the degree of curvature of a contour or the angle of the junction between two cylinders. MPs vary continuously with orientation in depth. The Gabor jet measure is only sensitive to MP variation and does not reflect the markedly greater sensitivity to NAP differences or to learned differences, such as that reflected in PDCs for faces. People (and macaques) find it exceedingly difficult to individuate an object that differs from foils only metrically when the object is encountered at a new orientation in depth, as documented by the difficulty in matching wire frame objects resembling bent paper clips that were studied extensively in the 1980s and 1990s. ${ }^{26,30}$ Cells in the inferior temporal region of the macaque modulate their firing much more to equal Gabor-jet image changes in a NAP, for example, from straight to curved, than changes in an MP, such as degree of curvature or differences in the angle of attachment of a pair of cylinders, suggesting a fundamental neural basis to the greater sensitivity to NAP compared to MP differences. ${ }^{31}$

This phenomenon of greater nonaccidental than metric sensitivity in face recognition is underscored in the striking demonstrations of Sinha and Poggio 32,33 in which observers fail to notice the substitution of a President's inner face for the inner face of his vice president (Clinton and Gore; Bush and Cheney). In these instances, the inner facial features vary metrically and subtly between the president and his vice-president and are dominated by the 
larger qualitative, i.e., nonaccidental, differences in the external configuration of hairline and head shape as well as eyeglasses, and context.

The absence in our face stimuli of surface features or variations of face attributes that differed nonaccidentally were motivated by the desire to limit the variation between faces to metric properties. As noted previously, with pairs of faces that differ in local nonaccidental features, observers will focus on the region with the featural difference and thus circumvent normal face processing. Normal face processing is characterized by configural effects, produced by the overlap of large receptive fields (RFs) which are centered at different positions on the face. ${ }^{28}$ They allow the face to be processed as a whole with the RF overlap allowing multiple redundant coding of the small metric differences between similar faces.

The present minimal match-to-sample paradigm can and employed to study the discrimination of any pair of stimuli, including metric variations of the harmonics of a sphere which produce smooth complex sculptured volumes resembling teeth. ${ }^{12}$ These have been employed as non-face comparison stimuli for studying what might be unique about faces. They show the same high correlations (in the mid -.90s) with RTs and error rates as the discrimination of faces.

Matching computer-generated vs. photos of faces. The present investigation employed computer generated (CG) images of faces. Crookes et al. ${ }^{30}$ showed that the "other race effect," a reduction in accuracy of face recognition when judging faces other than that of the participant's own race, was diminished with CG faces compared to photographs. It is possible that CG faces more generally show reduced sensitivity to PDCs, such as race, perhaps partly a function of their reduced animacy (noted by Crookes et al.), but it would seem implausible that the discrimination of physical variations within a single population classification, as in the present paradigm, could be any more sensitive to the physical variations as assessed by the near ceiling correlations with Gabor similarity ${ }^{12}$--without even any correction for the underlying unreliability of the behavioral data. This said, generalizations of results with CG faces need to be assessed with photographs despite the challenges of achieving control of local features and subtle differences in population-defined attributes. 
A subjective yardstick for orientation disparity effects on Gabor dissimilarity values. The present investigation demonstrated that modest orientation disparities produced large metric dissimilarities in images of faces. Figure 5 furnishes a subjective yardstick as to the extent at which a $20^{\circ}$ orientation disparity between two (otherwise identical) computer generated faces (left panel) approximate the dissimilarity between photos of the faces of two individuals with metric variations of their faces at the same $\left(0^{\circ}\right)$ frontal orientation but also differing greatly in the population-defined classifications of sex, race, expression, and facial hair (Figure 5, right panel). The Gabor dissimilarity values reflect only multiscale and multiorientation image (contrast) differences. They yield no greater weights to differences in NAPs or PDCs, strongly implying that the markedly greater sensitivity to these qualitative characteristics are a function of classifiers other than those engaged when distinguishing within population-defined differences in the absence of distinguishing NAPs. As discussed earlier in the plight of the prosopagnosic, the core challenge in face recognition is in individuating a face within a PDC, not in distinguishing among PDCs. The large costs in RTs and error rates in matching unfamiliar faces differing solely in orientation could thus be attributable to the heretofore unappreciated sizable increase in the dissimilarity of facial images produced by orientation disparities. When we encounter new individuals, we store the images of their faces at their various orientations for subsequent recognition which is why the large costs of depth-rotated facial images are only apparent with unfamiliar faces ${ }^{9}$.
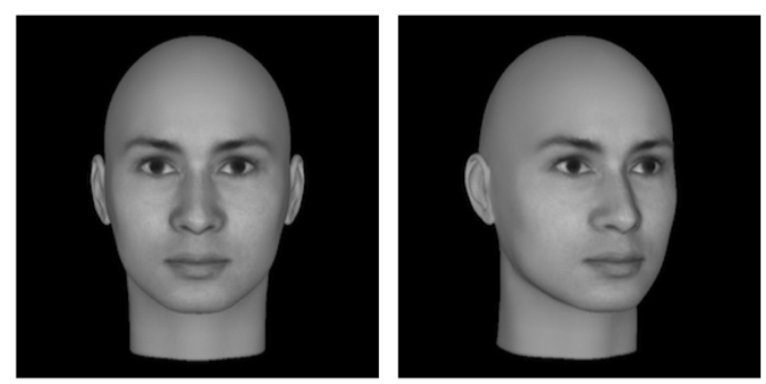

Gabor Dissim = 5.61
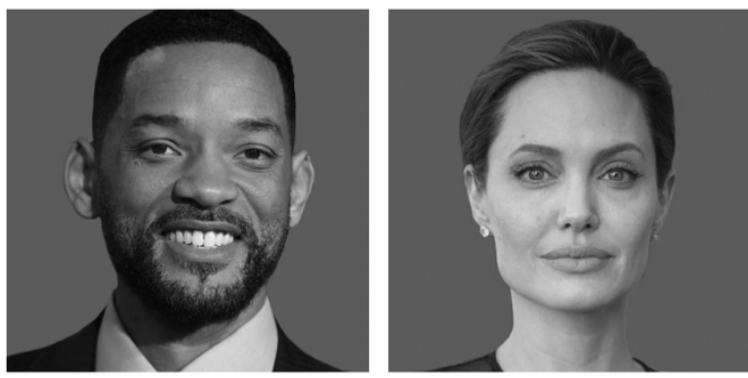

Gabor Dissim = 5.81

Figure 5. The dissimilarity between two of the experimental faces of the same identity with an orientation disparity of $20^{\circ}$ (left panel) is almost as large as two faces (right panel), at the same orientation, but differing in their PDCs of sex, race, expression, and facial hair, in addition to their variation within their own PDCs. Right panel, Getty Images.com. 


\section{REFERENCES}

[1] Duchaine, B., \& Nakayama, K. The Cambridge Face Memory Test: Results for neurologically intact individuals and an investigation of its validity using inverted face stimuli and prosopagnosic participants. Neuropsychologia, 44, 576-585 (2006).

[2] Bruce, V., Henderson, Z., Greenwood, K., Hancock, P. J. B., Burton, A. M., \& Miller, P. Verification of face identities from images captured on video. J. of Exp. Psychol.: Applied, 5, 339-360 (1999).

[3] Biederman, I., \& Kalocsai, P. Neurocomputational bases of object and face recognition. Phil. Trans. Royal Soc. London: Biol. Sci., 352, 1203-1219 (1997).

[4] Hill, H., Schyns, P., \& Akamatsu, S. Information and viewpoint dependence in face recognition. Cognition, 62, 201-222 (1997).

[5] Troje, N. F., \& Bülthoff, H. H. Face recognition under varying poses: The role of texture and shape. Vis. Res., 36, 1761-1771 (1996).

[6] Valentin, D., Abdi, H., \& Edelman, B. What Represents a Face? A Computational Approach for the Integration of Physiological and Psychological Data. Perception, 26, 1271-1288 (1997).

[7] Hancock, P. J. B., Bruce, V., \& Burton, A. M. Recognition of unfamiliar faces. Trends in Cognitive Sciences, 4, 330-337 (2000).

[8] Barense, M. D., Henson, R. N., Lee, A. C., Grahm, K. S. Medial temporal lobe activity during complex discrimination of faces, objects, and scenes: Effects of viewpoint. Hippocampus, 20, 389-401 (2010).

[9] Natu, V. S., \& O'Toole, A. J. Spatiotemporal changes in neural response patterns to faces varying in visual familiarity. Neuroimage, 108, 151-159 (2015).

[10] Lades, M., Vorbruggen, J., Buhmann, J., Lange, J., Malsburg, C. V., Wurtz, R., \& Konen, W. Distortion invariant object recognition in the dynamic link architecture. IEEE Trans. Comp., 42, 300-311 (1993).

[11] Margalit, E., Biederman, I., Herald, S. B., Yue, X., \& von der Malsburg, C. An applet for the Gabor scaling of the differences between complex stimuli. Attention, Perception, \& Psychophysics. 78, 2298-2306 (2016). 
[12] Yue, X., Biederman, I., Mangini, M. C., Malsburg, C. V., \& Amir, O. Predicting the psychophysical similarity of faces and non-face complex shapes by image-based measures. Vis. Res., 55, 41-46 (2012).

[13] Yue, X., Tjan, B. S., \& Biederman, I. What makes faces special? Vis. Res., 46, 3802-3811. (2006).

[14] Kanwisher, N. \& Yovel, G. The fusiform face area: A cortical region specialized for the perception of faces. Phil. Trans. Royal Soc. London: Biol. Sci., B. 361, 2109-28. (2006).

[15] Grill-Spector, K, Knouf, N. \& Kanwisher, N. The fusiform face area subserves face perception, not generic within-category identification. Nat. Neurosci., 7, 555-562. (2004).

[16] Troje, N. F., \& Bülthoff, H. H. How is bilateral symmetry of human faces used for recognition of novel views? Vis. Res., 38, 79-89. (1998).

[17] Mangini, M. C., \& Biederman, I. Making the ineffable explicit: Estimating the information employed for face classification. Cognitive Sci., 28, 209-226. (2004).

[18] Xu, X., \& Biederman, I. Neural correlates of face detection. Cerebral Cortex, 24, 1555-1564. (2014).

[19] Wiskott, L., Fellous, J. M., Krüger, N., \& von der Malsburg, C. Face recognition by elastic bunch graph matching. IEEE Trans. Pattern Anal. Mach. Intel., 19, 775-779. (1997).

[20] Müeller, M. K. \& Wuertz, R. P. Learning from Examples to Generalize over Pose and Illumination. In C. Alippi et al., Artificial Neural Networks, ICANN 2009, Part II, LNCS 5769, 643-652., Berlin Springer-Verlab. (2009).

[21] Richardson, J. T. E. Eta squared and partial eta squared as measures of effect size in educational research. Educat. Res. Rev., 6, 135-147. (2011).

[22] Townsend, J. T., \& Ashby, F. G. The Stochastic Modeling of Elementary Psychological Processes. Cambridge Univ. Press. (1983).

[23] Sternberg, S. The discovery of processing stages: extensions of Donders' method. Acta Psychologia Attention Performance, 30, 276-315. (1969).

[24] Sternberg, S. Modular processes in mind and brain. Cognitive Neuropsychology, 28, 3-4. (2011). 
[25] Biederman, I., \& Bar, M. One-shot viewpoint invariance in matching novel objects. Vis. Res., 39, 2885-2899. (1999).

[26] Biederman, I. Recognizing depth-rotated objects: A review of recent research and theory. Spat. Vis., 13, 241-253. (2001).

[27] Amir, O., Biederman, I., \& Hayworth, K. J. Sensitivity to nonaccidental properties across various shape dimensions. Vis. Res., 72, 35-53. (2012).

[28] Xu, X., Biederman, I., \& Shah, M. S. A neurocomputational account of the face configural effect. J. Vis., 14, 1-9. (2014).

[29] Crookes, K., Ewing, L., Gildenhuys, J., Kloth, N., Hayward, W. G., Oxner, M., Pond, S., \& Rhodes, G. How well do computer-generated faces tap face expertise? PLoS One 10: e0141353. doi:10.1371/journal.pone.0141353. (2015).

[30] Logothetis, N. K., Pauls, J., Bulthoff, H. H., \& Poggio, T. View-dependent object recognition by monkeys. Curr. Biol. 4. 401-414. (1994).

[31] Sinha, P., \& Poggio, T. I think I know that face .... Nature, 384/6608.404. (1996).

[32] Sinha, P., \& Poggio, T. United we stand: The role of head-structure in face recognition. Percept., 31, 133. (2002).

The authors declare no financial or non-financial competing interests.

Supported by the Dornsife Research Fund.

Open Practice Statement: The data have been deposited in The Open Science Framework, osf.io.

\section{Author Contributions}

$\mathrm{CMH}$ : Contributed to the theoretical development of study; performed advanced data analyses and scaling of stimuli; Created graphs and figures; Contributed to the literature review; participated in the writing.

IB: Conceived of and designed the study; developed the theory and guided the data analyses; primary author of manuscript. 
TZ: Supervised data collection; Organized data; Created graphs; Participated in pilot work. MN: Created stimuli; Ran subjects; Performed early data analyses.

EXM: Participated in early phases of study; organized data; performed data analyses and fiducial point scaling of the stimuli. 